\title{
Attitudes towards prescribing psychiatric medicines: do the views of psychology and medical students differ?
}

\author{
Carlos De las Cuevas ${ }^{1,2 *}$, Wenceslao Peñate ${ }^{3}$, Lilisbeth Perestelo ${ }^{2,4}$, Amado Rivero ${ }^{5}$, Jeanette Pérez ${ }^{5}$, \\ Marien González ${ }^{5}$, Alia Pérez-Wehbe ${ }^{5}$
}

${ }^{1}$ Department of Psychiatry, University of La Laguna, San Cristóbal de La Laguna, Canary Islands, Spain;
${ }^{2}$ CIBER en Epidemiología y Salud Pública (CIBERESP), Santa Cruz de Tenerife, Spain;
${ }^{3}$ Department of Personality, Assessment and Psychological Treatments, University of La Laguna, Canary Islands, Spain;
${ }^{4}$ Evaluation Unit of the Canary Islands Health Service (SESCS), Santa Cruz de Tenerife, Spain;
${ }^{5}$ Fundación Canaria de Investigación y Salud (FUNCIS), Santa Cruz de Tenerife, Spain.
Email: ${ }^{*}$ cdelascuevas@gmail.com

Received 1 July 2011; revised 12 August 2011; accepted 22 August 2011.

\begin{abstract}
Concordance has been suggested as a process of the consultation in which prescribing process is based on partnership. The aim of this cross-sectional study was to compare attitudes towards psychiatric medication and concordance in medicine taking between medical and psychology students, as they represent future members of mental health teams. Two hundred and sixteen medical students and 222 psychology students completed the Leeds Attitudes toward Concordance scale (LATCon) and the Beliefs about Medication Questionnaire (BMQ) (both adapted for psychiatric medication). Psychology students saw psychiatric medicines as more harmful and were also most likely to believe that psychiatrists overprescribed these medicines. They also scored higher than medical students on attitudes towards concordance, although this difference remained at the limit of statistical significance.
\end{abstract}

Keywords: Leeds Attitude toward Concordance Scale (LATCon); Beliefs about Medication Questionnaire (BMQ); Medical Students; Psychology Students

\section{INTRODUCTION}

Although there are slight variations in the structure and composition, multidisciplinary Community Mental Health Teams are considered to provide the core of Specialist Mental Health Services in Spain. They are responsible for delivering and for coordinating a specialized level of community-based care for defined populations [1]. In this context, psychiatrists and psychologists have to work together sharing approaches and objectives although they have obvious different skills arising from different professional backgrounds and different basic training.

Even though psychological treatments of proven efficacy are available for the management of many psychopathological disorders, the prescription of psychoactive medication is one of the most common interventions in mental healthcare. However, the gap between best care and usual care is large for psychiatric disorders. In particular, poor adherence to treatment of psychiatric disorders is a worldwide problem of striking magnitude that contributes to the gap care [2]. Poor adherence to longterm therapies compromises severely the effectiveness of treatment, making this a critical issue in population health from the perspective of both quality of life and health economics [3]. This lack of adherence is not only a problem of pharmacotherapy, but also a problem in psychological treatments [4].

The ability of patients to follow treatment plans in an optimal manner is frequently compromised by diverse barriers, usually related to different aspects of the problem that include: social and economic factors, the health care team/system, the characteristics of the disease, disease therapies and patient-related factors. Solving the problems related to each of these factors is necessary if patients' adherence to therapies is to be improved. In this sense, experts recommended, especially, to devote time in treatment specifically in addressing medication adherence, assess patients' motivation to take prescribed medications, and to focus on strengthening the therapeutic alliance [2]. This implies some changes in the traditional model of the health service delivery.

The traditional model of the practitioner-patient interaction incorporates a practitioner-centred approach, focusing on the disease rather than on the patient. The "the- 
rapeutic alliance" represents another point of view, and it is based on the philosophy of "concordance" (or "partnership in medicine-taking"). Concordance is a psychological interaction process that puts the patient at the centre of the relationship, interacting reciprocally with the practitioner. In the concordance model, patients are viewed as active participants who manage their own health care. Practitioners and patients are encouraged to forge partnerships to work together as equals. Practitioners bring their professional expertise to the table, whilst patients draw on their own experiences, beliefs and wishes [5]. Concordance is viewed as a strategy to improve medication adherence, among other complex interventions [6].

The problem of treatment adherence is especially relevant in mental health services, since psychiatric patients are more likely than others to refuse medication [7], and are more likely to participate in therapeutic decisions [8]. The importance of (non)adherence in mental health practice has dealed to the creation of a expert panel to afford potential solutions for adherence [9]. A prior consensus among healthcare practitioners is the basic target to get those potential solutions.

Psychiatrists and clinical psychologists are part of the healthcare team that has daily contact with patients to discuss medication. If they have different respective beliefs about psychoactive medication, what treatment a patient should receive and the consequent provision of different information to the patient about the treatment, the patient could then have difficulties evaluating the treatment [10]. Thus, agreement between mental health professionals is a pre-requisite in treatment election and patient's commitment. This includes agreement in psychiatric medication and concordance. This is a relevant aim, since there are different perceptions about medicine depending on the different educational background of practitioners [11]. Psychiatrists and clinical psychologists have different formative curricula in both their theoretical and practical formation, and it can be part of a lack of consensus about mental health intervention.

The objective of the present paper is to investigate the beliefs of medical and psychology students about psychiatric medication and their attitudes towards concordance in medicine taking, as they potentially represent future mental health care providers. Potential differences in attitudes towards prescribing psychiatric medicines between psychiatrists and clinical psychologists could originate from their different academic formation which could favor a biological perspective in the case of medical students and a more psychosocial view for psychology students. Therefore, we assume that medical students will show more positive beliefs about psychiatric medicines, while psychology student will show a better attitude towards concordance.

\section{METHODS}

\subsection{Participants}

216 medical students and 222 psychology students participated in the study. According to their level degrees, medicine students were 63 third degree, 57 fourth degree, 56 fifth degree, and 40 sixth degree. Psychology students: 85 third degree, 69 fourth degree, 46 fifth degree, and 22 master degree. Medicine students mean age was 22,62; psychology students mean age was 22.2. Based on sex, medical students: 64 were males, and 152 females; psychology students: 41 males, and 181 females.

Students were recruited during class lessons. They were asked whether they want to voluntarily participate in a study about mental health treatment. There was not any credit nor specific remuneration for their participation.

\subsection{Instruments and Measures}

In order to measure students' beliefs about psychiatric medication, the Spanish version of BMQ-General questionnaire was used. The scale assesses general beliefs or social representations of pharmaceuticals as a type of treatment: beliefs that medicines in general are overused by doctors and beliefs that medicines in general are harmful, addictive, poisons that should not be taken continuously. BMQ have shown to be a valid and reliable measure [12-14] and it is able to discriminate between different groups of patients and students [11-14]. The BMQ-General scale includes eight items in two subscales, the Overuse subscale and the Harm subscale. The degree of agreement with each statement is indicated on a five point Likert scale, ranging from (1) strongly disagree to (5) strongly agree. For this study, items were modified to specifically refer to psychiatric medication.

In order to investigate the attitudes of medical and psychology students towards concordance, the Leeds Attitude toward Concordance Scale (LATCon) was used $[15,16]$. It consists of a 12 -item scale. The respondent scores each item on a four point Likert scale: (0) strongly disagree, (1) disagree, (2) agree, (3) strongly agree. The total maximum score is therefore 36 . An average item score between 2 and 3 indicates that the respondent tends to 'agree' with the concept of concordance, while an average score below 2 suggests that he/ she does not.

The original English version of the questionnaire was translated into Spanish by two members of the research team and then translated back into English by a native English speaker to check whether or not the Spanish translations conveyed the original meaning intended by the authors. As with the BMQ, items were modified to specifically refer to psychiatric medication. 
The questionnaire that the medical and psychology undergraduate students completed also included some sociodemographic variables and information about present or past use of psychoactive drugs by the interviewee or their relatives.

\subsection{Study Design}

According to Montero and León [17], a post-facto design was used. This was a cross-sectional, self-administered questionnaire survey of an opportunistic sample of medical and psychology students registered in the last courses of their respective degrees as undergraduates at the University of La Laguna, in the Canary Islands, Spain.

\subsection{Data Collection}

The data collection for this cross-sectional study was performed in May 2010, during class lessons. At the time of the data collection students were informed both orally and in writing about the aim of the study and that participation was voluntary and anonymous. They also received information on how to complete the questionnaires.

\subsection{Data Analysis}

Mean differences between medical and psychology students were calculated using one way MANOVA (BMQ subscales) and t-test (LATCon). For BMQ subscales, the effect of having personal or vicarious experience with psychiatric drugs, as well as its interaction with type of studies, were analyzed.

Apart from total and factor-scores comparisons, an item-level analysis was also carried out, by means of two additional MANOVAs with BMQ and LATCon items, respectively, as dependent variables. Finally, a forward stepwise logistic regression was conducted with both the BMQ-General and LATCon items as independent variables, and type of studies as dependent variable.

\section{RESULTS}

\subsection{Sample}

There was no significant difference in both age and level degree between student groups. Psychology students included significantly more women $(81.5 \%$ vs. $70.4 \%$; $\left.\chi^{2}=7.483, \mathrm{p}<0.006\right)$.

\subsection{Beliefs about Medications}

Scores for the BMQ Overuse and Harm subscales were normally distributed in both samples. No significant differences in general beliefs about medication were found due to age or sex for any student category considered. According to BMQ subscales scores, psychology students saw psychiatric medicines as more harmful compared to medical students (BMQ-Harm: $2.68 \pm 0.58$ vs. $2.38 \pm 0.62 ; \mathrm{p}<0.001)$ and were also most likely to believe that psychiatrists overprescribed these medicines (BMQ-Overuse: $3.67 \pm 0.66$ vs. $3.18 \pm 0.7 ; p<0.001$ ).

Table 1 shows the mean scores in the BMQ items for medical and psychology students. Taking as a criterion a theoretical intermediate score of 3 for each item (neither agree nor disagree), both medical and psychology students surpassed this score on items related to the addictive nature of the psychiatric medication and the consid-eration that spending more time with patients would result in less prescribed medication. There are two other

Table 1. Differences in BMQ items for medical and psychology students (MANOVA).

\begin{tabular}{|c|c|c|c|c|c|}
\hline \multicolumn{6}{|l|}{ Beliefs About Medicines Questionnaire-General Items } \\
\hline Overuse subscale & $\begin{array}{l}\text { Medicine Mean } \\
\text { (SD) }\end{array}$ & $\begin{array}{l}\text { Psychology } \\
\text { Mean (SD) }\end{array}$ & $\mathbf{F}$ & $\mathbf{p}$ & $\eta^{2}$ \\
\hline 1. Psychiatrists use too many medicines & $2.98(0.85)$ & $3.67(0.85)$ & 70.635 & 0.000 & 0.139 \\
\hline 7. Psychiatrists place too much trust on medicines & $2.98(1.01)$ & $3.70(0.75)$ & 71.132 & 0.000 & 0.140 \\
\hline 8. If psychiatrists had more time with patients they would prescribe fewer medicines & $3.61(1.09)$ & $3.65(0.98)$ & 0.181 & 0.670 & 0.000 \\
\hline \multicolumn{6}{|l|}{ Harm subscale } \\
\hline $\begin{array}{l}\text { 2. People who take psychiatric medicines should stop their treatment for a while every } \\
\text { now and again }\end{array}$ & $2.64(1.12)$ & $2.95(1.02)$ & 9.473 & 0.002 & 0.021 \\
\hline 3. Most psychiatric medicines are addictive & $3.15(1.13)$ & $3.47(0.98)$ & 9.804 & 0.002 & 0.022 \\
\hline 4. Natural remedies are safer than psychotropic medicines* & $2.36(1.02)$ & $2.72(0.99)$ & 13.650 & 0.000 & 0.030 \\
\hline 5. Psychiatric medicines do more harm than good & $1.96(0.79)$ & $2.30(0.77)$ & 20.695 & 0.000 & 0.045 \\
\hline 6. All psychiatric medicines are poisons & $1.81(0.98)$ & $1.96(0.96)$ & 2.601 & 0.108 & 0.006 \\
\hline
\end{tabular}

*In the Spanish validation of BMQ, item 4 loads on Harm subscale, contrary to the original questionnaire. 
items in which psychology students scored above this term: the facts that psychiatrists use too many medicines and that they place too much trust on their prescriptions. These data indicate that, overall, the two samples tend to disagree more than to agree with the contents of the items of BMQ-General scale.

As shown in Table 1, six out of the eight items Registered significant differences with the psychology students scoring higher than the medical students: they were most likely to believe that patients should discontinue the use of drug from time to time, that psychiatric drugs are addictive, and that these compounds do more harm than good or that natural remedies are more useful. However, considering the effect size $\left(\eta^{2}\right)$, the differences are really low. Only the differences found in the items about overuse and excessive reliance on psychiatric drugs seem to have reached a certain consistency. On the other hand, no significant differences were registered in the item that considers psychiatric medications as poisons and the already mentioned that if psychiatrists had more time with patients they would prescribe fewer medicines.

Having in mind that a greater knowledge of psychoactive drugs might influence the student's beliefs, the sample was divided into two groups: those who have been treated with such compounds or that have a relative or close friend under this treatment $(n=240)$ and those who have no experience on these drugs $(n=198)$. The only significant item was the one corresponding to "all psychiatric medications are poisons" (F1, $436=5.52 ; \mathrm{p}$ $\left.\leq 0.019 ; \quad \eta^{2}=0.013\right)$ with the group without experience about $(\mathrm{M}=2.01$; $\mathrm{SD}=0.95)$ considering the psychoactive drugs more toxic than the group who knew them (M $=1.79 ; \mathrm{SD}=0.97)$. The interaction between this variable and the type of studies only found in one item and it was marginally significant $\left(\mathrm{F} 1,434=3.83 ; \mathrm{p} \leq 0.051 ; \quad \eta^{2}=\right.$ 0.01 ), suggesting that psychology students have a greater tendency to disregard toxic psychotropic drugs when they know them.

\subsection{Attitude to Concordance}

Considering the results of the Leeds Attitude to Concordance Scale (LATCon), the mean item score of the global sample was $2.00 \pm 0.35$, median score 2 (Table 2). These values indicate that the respondents tend to "agree" with the concept of concordance. There was no difference in the attitudes towards concordance of the medical and psychology students (mean item score: medicine $=1.97$ \pm 0.33 ; psychology $=2.03 \pm 0.37 ; \mathrm{p}=0.07$ ).

In the comparison of items means across the two samples, five items allow to appreciate differences between medical and psychology students. In four of the items

Table 2. Differences in LATCon items for medical and psychology students (MANOVA).

Leeds Attitude to Concordance Scale-items
Medicine Mean Psychology Mean

(SD)

$1.52(0.71)$

$2.22(0.61)$

$2.13(0.62)$

$1.51(0.93)$

$2.08(0.72)$

$2.56(0.58)$

$2.18(0.70)$

$2.07(0.65)$

$2.46(0.60)$

$1.10(0.76)$

$1.89(0.59)$

$1.94(0.60)$
(SD)

F

p $\quad \eta^{2}$

p $\quad \eta^{2}$

1. The consultation between the psychiatrist and patient should be viewed as a

2. Psychiatrists should respect their patients' personal beliefs \& how they cope

achieve

medication taking an experiment carried out by the patient

5. Psychiatrists should give patients the opportunity to talk about their thoughts

6. Better health would follow from co-operation between psychiatrists and patients

7. A high priority in the consultation between psychiatrist and patients is to establish agreement about the need for medicine

8. Psychiatrists should be sensitive to patient desires, needs and abilities

9. Psychiatrists should try to help patients to make as informed a choice as possible about benefits and risks of alternative treatments

10. During the psychiatrist-patient consultation, it is the patient's decision that is most important

11. Psychiatrists should be more sensitive to how patients react to the information they give

12. Psychiatrists should try to learn about the beliefs their patients hold about their medicines

$\begin{array}{llll}1.71(0.75) & 7.631 & 0.006 & 0.017 \\ 2.19(0.64) & 0.228 & 0.633 & 0.001 \\ 2.11(0.65) & 0.126 & 0.723 & 0.000 \\ 1.42(0.86) & 1.122 & 0.290 & 0.003 \\ 2.07(0.72) & 0.052 & 0.820 & 0.000 \\ 2.50(0.52) & 1.323 & 0.251 & 0.003 \\ 2.05(0.67) & 4.265 & 0.039 & 0.010 \\ 2.23(0.64) & 6.842 & 0.009 & 0.015 \\ 2.49(0.59) & 0.244 & 0.622 & 0.001 \\ 1.48(0.77) & 26.605 & 0.000 & 0.058 \\ 2.22(0.54) & 36.835 & 0.000 & 0.078 \\ 1.94(0.68) & 0.001 & 0.977 & 0.000\end{array}$


(the consultation is a negotiation between equals; doctors should be sensitive to patients desires, needs, abilities and how patients react to the information they give; and the patient decision is the most important), psychology students scored significantly higher than those of medicine. However, with regard to the priority to establish agreement about the need for medicines, the medical students were the ones who scored higher. In eight out of 12 items composing the scale, both groups scored above 2 (indicating agreement rather than disagreement). In four of the items both samples disagree with the assertion. They included the consideration that the consultation between doctor and patient should be viewed as a negotiation between equals, the fact that the patients' decision is the most important one, the experimental nature of prescribing and using psychoactive drugs, and the need for psychiatrists to learn about the beliefs their patients hold about their medication.

Finally, stepwise logistic regression (Wald forward method) was carried out to try to identify which items of the BMQ and LATCon scales differ for medical and psychology students. The final resulting model included 10 items, with a Nagelkerke $\mathrm{R}^{2}=0.43$, highly significant $\left(x^{2}=171.72 ; \mathrm{p} \leq 0.001\right)$. The Table 3 summarizes the model. As it can be seen, the variables that best predict are the BMQ items pertaining to the Overuse subscale. Six items about concordance become part of the equation with secondary importance, with significant coeffi- cients, but with a low incremental weight (with the exception of the consideration that psychiatrists should be more sensitive to information provided by the patient).

\section{DISCUSSION}

The improvement of health services includes the need of an agreement among health teams about therapeutic process. Attitudes about medicines, and about the concordance between patients and practitioners, are parts of that agreement. This is especially relevant in mental health services, because of there are different practitioners with different curricula (i.e., psychiatrics, psychologists...), and because mental patients are more sensitive about their treatments. Additionally, despite some social changes, a stigma of mental disorders still prevents patients from seeking help [18].

According to our results, the first consideration to highlight is that, in general, medical and psychology students agree in that medication is not always the best choice and that concordance between patient and therapist is a value to keep in mind within the therapeutic relationship. Somehow this information indicates that both groups are more in agreement rather than in disagreement. The necessary coordination between these two professional groups in the attention they pay to mental health seems to have certain guarantees, though some differences persist. The reason for this does not seem to be justified by the academic curriculum or the

Table 3. Forward stepwise logistic regression with BMQ and LATCon items as independent variables and type of student as dependent variable (medical students categorized as 0 ).

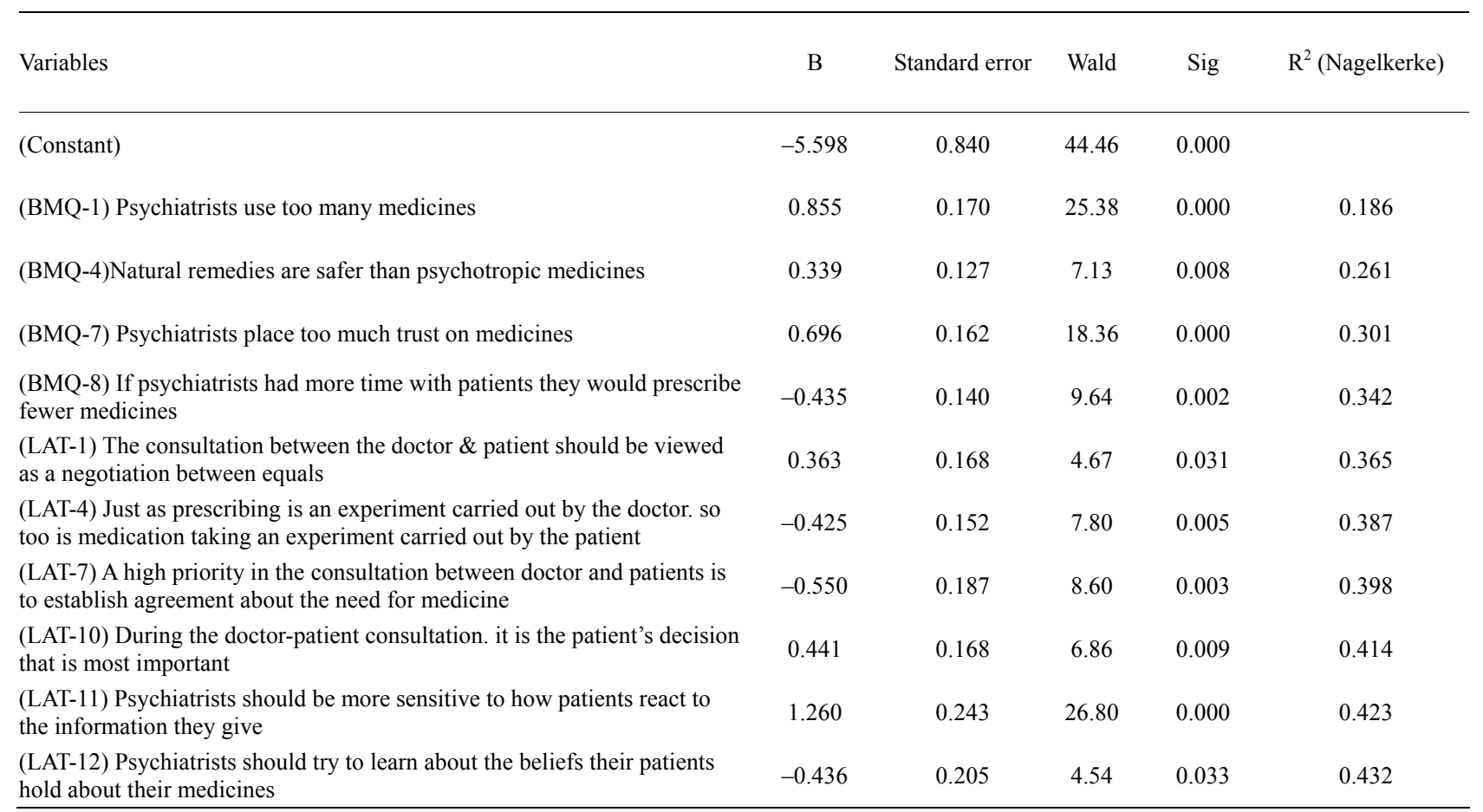


training reasons, as the academic contents of these studies are uneven. It is possible that other factors are influencing this, such as the social and human values (the less medication the better, a good patient-therapist relationship is better than a bad relation), or the social desirability ("politically correct"). This is especially relevant in the case of the LATCon, provided that both students groups highly agree in two thirds of the items.

Analyzing the differences and differential profile found, it is striking that the major weight rests on medication rather than on conformity. In this case, it can be due to the training curriculum, because of the fact that in psychology the functional factors are accentuated as the pathology's determinant elements, opposite to the more prominent biological component in medicine. On the other hand, culture favors a vision of the medication as an unquestionable healer (and as such it is a 'professional' decision). This element would be more deeply rooted in medicine students that in those of psychology due to the proximity to this cultural tradition. Our data point out that the modulating function of knowing the psychoactive drugs' effects does not seem to play a relevant role. Nevertheless, the necessary patient-therapist relationship passes to be of secondary importance, possibly because both student groups have a similar social value on mutual commitment, and on the need of empathy between patient and physician. In any case, some differences could be observed, showing overall that psychology students showed a higher agreement on the importance of concordance.

These differences can be justified by the epistemological diversity in the medical and psychological treatments' nature. In psychotherapy there is an assertion which calls for an active patients' participation in health change. On the other hand, in medicine this assertion is mediated by the intrinsic treatments' effects (especially medication taken). In other words, concordance is part of an active principle of psychological treatments, but in medicine it is part of a better procedure (better adherence, better commitment). In this sense, psychologists encourage patient-therapist concordance as an intrinsic element of treatment efficacy, whereas physicians consider it as a modulator variable, via adherence, of treatment efficacy.

Definitively, medical and psychology students seem to show a raising agreement in the need of concordance within the therapist-patient relationship. Taking into account the training differences [19] the fact that the agreement is major for both professional groups it might be indicating the need of new contents that trace these training differences in relation with concordance. In this respect, new versions of the LATCon could favor this point of view [20]. In any case, beyond new versions of the LATCon could improve its differential and discriminant validity, it might be more appropriate to bear in mind the major impact that the levels of social desirability have on concordance when it is always seen as "what it should be".

The most notable differences were found in the role of medication and its management. Beyond the modulating paper of social and personal values of every students group, the training curriculum could be modulating these results; for this reason it would be necessary to consider the role of the curricular development [21] and that of the professional practice (clinical psychology and psychiatry) as elements that could go eliminating these differences. This does not mean that both curricula must share more equivalent contents between them, and more equivalent professional responsibilities, as the polemic of prescription privileges for clinical psychologists seems to point out [22]. We do not find reasons to confuse both the functional and biological perspectives in mental health. But we find reasons for a better complementary formation in clinical practice. As it has been mentioned, the necessary complementarity and conformity among mental health professionals makes it necessary that the elimination of these differences is tackled explicitly. The agreement between professionals who shared a common task is a prerequisite of its efficacy and efficiency.

It is important to point out, as a main limitation of this study, that only a small proportion of medical and psychology undergraduates go on to work in the area of mental health, so these results are not directly generalizable to future psychiatrists and clinical psychologists. Nevertheless, they offer a general view of differences in attitude towards psychiatrist medicines and concordance between these student samples, differences that therefore could be present independently of the eventual academic education and training in mental health area.

\section{ACKNOWLEDGEMENTS}

This work was supported by the Instituto de Salud Carlos III, FEDER Unión Europea (PI10/00955).

\section{REFERENCES}

[1] Gómez-Beneito, M. (2009) Evaluación (y actualización) de la estrategia en Salud Mental del sistema nacional de Salud. Available at: 2009, Accessed on July 7, 2010. http://www.ascane.es/Informe-de-Evaluacion_Salud Mental_CISNS.pdf

[2] Julius, R.J., Novitsky, M.A. Jr. and Dubin, W.R. (2009) Medication adherence: A review of the literature and implications for clinical practice. Journal of Psychiatric Practice, 15, 34-44.

doi:10.1097/01.pra.0000344917.43780.77 
[3] Sabaté, E. (2003). Adherence to long-term therapies evidence for action. World Health Organization, Geneva.

[4] Echeburúa, E., Sarasua, B., Zubizarreta, I., Amor, P.J. and de Corral, P. (2010) Variables predictoras del rechazo, abandono y fracaso terapéutico en hombres violentos contra su pareja tratados psicológicamente en un marco comunitario. International Journal of Clinical and Health Psychology, 10, 403-420.

[5] Marinker, M. (1997) From compliance to concordance: Achieving shared goals in medicine taking. Royal Pharmaceutical Society/Merck Sharp \& Dohme, London.

[6] Haynes, R.B., Ackloo, E., Sahota, N., McDonald, H.P. and Yao, X. (2008) Interventions for enhancing medication adherence. Cochrane Database Systematic Review, 16, CD000011.

[7] Jónsdóttir, H., Friis, S., Horne, R., Pettersen, K.I., Reikvam, A. and Andreassen, O.A. (2009) Beliefs about medications: Measurement and relationship to adherence in patients with severe mental disorders. Acta Psychiatrica Scandinavica, 119, 78-84. doi:10.1111/j.1600-0447.2008.01279.x

[8] De las Cuevas, C., Rivero, A., Perestelo-Perez, L., Gonzalez, M., Perez, J. and Peñate, W. (2011, in press) Psychiatric patients' attitudes towards concordance and shared decision making. Patient Education and Counseling. doi:10.1016/j.pec.2011.02.015

[9] Velligan, D., Sajatovic, M., Valenstein, M., Riley, W.T., Safren, S., Lewis-Fernandez, R., Weiden, P., Ogedegbe, G. and Jamison, J. (2010) Methodological challenges in psychiatric treatment adherence research. Clinical Schizophrenia and Related Psychoses, 4, 74-91. doi:10.3371/CSRP.4.1.6

[10] Mardby, A., Akerlind, I. and Hedenrud, T. (2009) General beliefs about medicines among doctors and nurses in out-patient care: A cross-sectional study. BMC Family Practice, 10, 35. doi:10.1186/1471-2296-10-35

[11] Horne, R., Frost, S., Hankins, M. and Wright, S. (2001) In the eye of the beholder: Pharmacy students have more positive perceptions of medicines than students of other disciplines. International Journal of Pharmacy Practice, 9, 85-89. doi:10.1111/j.2042-7174.2001.tb01035.x

[12] Beléndez, M., Hernández, A., Horne, R. and Weinman, J. (2007) Evaluación de las creencias sobre el tratamiento: Validez y fiabilidad de la versión española del beliefs about medicines questionnaire. International Journal of Health \& Clinical Psychology, 7, 767-779.

[13] Horne, R. and Weinman, J. (1999) Patients' beliefs about prescribed medicines and their role in adherence to treatment in chronic physical illness. Journal of Psychosomatic Research, 47, 555-567.

doi:10.1016/S0022-3999(99)00057-4

[14] Mahler, C., Hermann, K., Horne, R., Jank, S., Haefeli, W.E. and Szecsenyi, J. (2011) Patients' Beliefs about Medicines in a primary care setting in Germany. Journal of Evaluation in Clinical Practice, in press. doi:10.1111/j.1365-2753.2010.01589.x

[15] Raynor, D.K., Thistlethwaite, J.E., Hart, K. and Knapp, P. (2001) Are health professionals ready for the new philosophy of concordance in medicine taking? International Journal of Pharmacy Practice, 9, 810-814. doi:10.1111/j.2042-7174.2001.tb01034.x

[16] Thistlethwaite, J.E., Raynor, D.K. and Knapp, P. (2003) Medical students' attitudes towards concordance in medicine taking: Exploring the impact of an educational intervention. Education for Health, 16, 307-317. doi:10.1080/13576280310001607424

[17] Montero, I. and León, O. (2007) A guide for naming research studies in Psychology. International Journal of Clinical and Health Psychology, 4, 505-520.

[18] Rojas-Vilches, A.P., Negy, C. and Reig-Ferrer, A. (2011) Attitudes toward seeking therapy among Puerto Rican and Cuban American young adults and their parents. International Journal of Clinical and Health Psychology, 11, 313-341.

[19] Weissman, M.M., Verdeli, H., Gameroff, M.J., Bledsoe, S.E., Betts, K., Mufson, L., Fitterling, H. and Wickramartne, P. (2006) National survey of psychotherapy training in psychiatry, psychology, and social work. Archives of General Psychiatry, 63, 925-934. doi:10.1001/archpsyc.63.8.925

[20] Knapp, P., Raynor, D.K., Thistlethwaite, J.E. and Jones, M.B. (2009) A questionnaire to measure health practitioners' attitudes to partnership in medicine taking: LAT Con II. Health Expectations, 12, 175-186. doi:10.1111/j.1369-7625.2009.00545.x

[21] Muse, M. and McGrath, R.E. (2010) Training comparison among three professions prescribing psychoactive medications: Psychiatric nurse practitioners, physicians, and pharmacologically trained psychologists. Journal of Clinical Psychology, 66, 96-103.

[22] Lavoie, K.L. and Barone, S. (2006) Prescription privileges for psychologists: A comprehensive review and critical analysis of current issues and controversies. CNS Drugs, 20, 51-66. doi:10.2165/00023210-200620010-00005 